\title{
The Effect of Requester Training on the Relevance of Lumbar Spine X-Ray Request for Low Back Pain in Adults at Yaounde
}

\author{
Moulion Tapouh Jean Roger1,2, Djomou Wandji Franck Arold3, Mbede Maggy3,4, Moifo Boniface ${ }^{3,5}$ \\ ${ }^{1}$ Bafoussam Regional Hospital Center, Bafoussam, Cameroon \\ ${ }^{2}$ Department of Radiology, Biophysics and Medical Imaging, Faculty of Medicine and Pharmaceutical Sciences, \\ University of Dschang, Dschang, Cameroon \\ ${ }^{3}$ Department of Medical Imaging and Radiation Oncology, Faculty of Medicine and Biomedical Sciences, University of Yaounde I, \\ Yaounde, Cameroon \\ ${ }^{4}$ Yaounde Central Hospital, Yaounde, Cameroon \\ ${ }^{5}$ Gynaeco-Obstetric and Pediatric Hospital of Yaounde, Yaounde, Cameroon \\ Email: tapouh@gmail.com
}

How to cite this paper: Roger, M.T.J., Arold, D.W.F., Maggy, M. and Boniface, M. (2022) The Effect of Requester Training on the Relevance of Lumbar Spine X-Ray Request for Low Back Pain in Adults at Yaounde. Open Journal of Radiology, 12, 8-16. https://doi.org/10.4236/ojrad.2022.121002

Received: December 7, 2021

Accepted: February 28, 2022

Published: March 3, 2022

Copyright $\odot 2022$ by author(s) and Scientific Research Publishing Inc. This work is licensed under the Creative Commons Attribution International License (CC BY 4.0).

http://creativecommons.org/licenses/by/4.0/

\section{(c) (i) Open Access}

\begin{abstract}
Background: Despite the availability of guidelines, the requests for medical imaging examinations for the exploration of low back pain remain irrelevant in the majority of cases. Objective: To assess the impact of requester training on the justification of lumbar spine X-rays for low back pain in adults in Yaounde. Materials and Methods: We carried out an interventional study from December 2017 to April 2018 in Yaounde (Cameroon). We analyzed the relevance of the prescription of X-rays for low back pain before and after an interactive training of the requesters. Results: Among the 16 requesters who were trained, the proportion of irrelevant requests dropped from $58.22 \%(46 / 79)$ before training to $44.44 \%(20 / 45)$ after training, representing a decrease of $13.78 \%(\mathrm{p}=0.012)$. The highest drop in the proportion of irrelevant requests was found among general practitioners $(-21.98 \%)$ and requesters having less than 10 years of experience in medical practice $(-31.8 \%)$. Conclusion: The training of requesters moderately reduces the rate of irrelevant examinations. Other factors of irrelevant prescription must be investigated and corrected for this decrease to be more significant.
\end{abstract}

\section{Keywords}

Guidelines, Low Back Pain, Training, Requesters, Justification

\section{Introduction}

Low back pain (LBP) is a major public health problem in the world because of its 
impact on the daily life of patients and its relatively high cost of care. It is one of the most frequent reasons for consultation in rheumatology and general medicine. In France, $60 \%$ to $90 \%$ of adults suffer or have suffered from LBP and its annual incidence in the adult population is estimated between $5 \%$ and $10 \%$ [1] [2]. In Cameroon, the hospital prevalence of chronic LBP is high. In a series of 583 patients seen in a rheumatology consultation at the Douala General Hospital in 2009 , Doualla et al. reported that $36.36 \%$ (212/583) suffered from LBP, including 197 patients who had experienced it for more than 3 months [3].

The use of imaging in the exploration of LBP is well codified by several guidelines, with lumbar X-ray being the first-line examination in most cases [4]. Unfortunately, these guidelines on the prescription of radiography in LBP are not always applied. In 2005, Webster et al. demonstrated that only $22.5 \%$ of requests for lumbar spine X-rays complied with recommendations for the management of common LBP in the United States [5]. In the same country, Pham et al. found in 2009 that the request for medical imaging examination was too early (before 28 days) in $28.8 \%$ of cases in elderly patients with LBP [6]. In Cameroon, noncompliance with recommendations for requesting medical imaging examinations is frequent. In a study carried out in Yaounde in 2013, Moifo et al. found that $23 \%$ of 262 examination requests (including standard radiography) were not relevant regarding the clinical information provided [7].

The low level of compliance with the guidelines could be linked to the ignorance of these guidelines by requesters alongside other factors such as conflicts of interest and difficulties in accessing appropriate health services. Good information for patients and training of requesters could significantly improve the justification of imaging requests for LBP [8] [9].

We carried out this study to assess the impact of requesters' training on the justification of lumbar spine X-ray requests for LBP in adults at Yaounde.

\section{Materials and Methods}

\subsection{Study Design and Study Site}

We carried out an interventional study in the medical imaging departments of 03 health facilities in Yaounde (capital city of Cameroon): The Yaounde Central Hospital, The Yaounde Gynaeco-Obstetric and Pediatric Hospital (YGOPH) and the Autonomous Center of Radiology and Medical Imaging (CARIM).

\subsection{Duration and Study Procedure}

The study lasted 5 months (from December 2017 until April 2018) and was subdivided into 3 phases:

- $1^{\text {st }}$ phase (December 2017 - February 2018): evaluation of the appropriateness of lumbar spine $\mathrm{X}$-ray requests through the patients examination and request form assessment;

- $2^{\text {nd }}$ phase (intervention): convenience selection of requesters representative of $60 \%$ of requests and their training by providing them the guidelines of the 
French Society of Radiology on the radiological management of LBP and discussing with them during a 45 minutes personalized explanation session;

- $3^{\text {rd }}$ phase (March 2018 - April 2018): second, evaluation of the appropriateness of lumbar spine X-ray requests and comparison with the results from the $1^{\text {st }}$ evaluation for requesters who have received the training.

\subsection{Study Population}

In this study, we included:

- Physicians who requested lumbar spine X-rays for LBP in adults living in Yaounde, who accepted to participate in the study and whose imaging requests represented at least $4 \%$ of all prescriptions before the intervention within the study period.

- Imaging requests written for LBP and patients who accepted to answer our questions.

The whole requesters and imaging requests fulfilling our inclusion criteria were selected for the study.

\subsection{Data of Interest}

We identified:

- Clinical information: circumstances leading to and duration of LBP, type of pain (mechanic or inflammatory), location and intensity of the pain, irradiation, number of episodes, triggers and alleviating factors, past medical history of the patient, signs associated with LBP, history of lumbar trauma, medical treatment received;

- The requester (physician or the patient himself);

- Requester details: qualification, area of exercise, number of years of experience.

These data were collected from imaging request forms, patient interrogation and interviews with the requesters.

Each lumbar spine X-ray was classified as "relevant" or "irrelevant". The relevance of the request was assessed according to the definition of the French Society of Radiology guidelines as follows:

- Relevant (correct): when its request is "Indicated", "Indicated only in particular cases", or "Specialized examination";

- Irrelevant (incorrect): when its request is "not indicated", "initially not indicated", or "contra-indicated" [9].

The appropriateness of X-rays was assessed simultaneously by a radiologist with 10 years of experience and a 7 th year medical student.

\subsection{Procedure of Data Collection}

The request forms were collected at the secretariat of each medical imaging department. Data from the radiology request forms and data from the patient interrogation were recorded on an anonymous survey form. 


\subsection{Intervention Procedure}

We selected requesters who frequently request for lumbar spine X-rays (at least $4 \%$ of the total requests) during the first phase of the study. Thereafter, we called for them to obtain their consent, booked an appointment during which we gave them the guidelines of the French Society of Radiology on LBP imaging in paper form (wall posters, individual summary sheets) and digital form (PDF file). At each meeting, we discussed during at least 45 minutes with the requesters to ensure a better understanding of the guidelines.

\subsection{Statistical Analysis}

Data were analyzed with the SPSS 22.0 software (Statistical Package for the Social Sciences).

The Chi-square test was used to measure the association between the proportion of request relevance and the qualification of the requester and the association between the request relevance and the number of years of experience of the requester.

The McNemar test was used to compare the proportion of relevant requests before and after the training with the aim to measure the impact of the intervention.

A p value $<0.05$ was considered as statistically significant.

\subsection{Ethical Considerations}

We obtained an ethical approval from the ethical committee of the Faculty of Medicine and Biomedical Sciences of the University of Yaounde I. In each of the three sites of recruitment, we obtained a research authorization from the Director of the institution. The patients and requesters informed consent were required before including them in the study. Information on individuals were treated with confidentiality.

\section{Results}

We collected 191 imaging requests of which 146 were collected before the intervention and 45 were collected after the intervention.

\subsection{Socio-Demographic Characteristics and Distributions of the Requesters}

Most of the requesters were specialist doctors (47.12\%) and clinicians with more than 15 years of experience (38.22\%) (Table 1 ).

\subsection{Relevance of X-Ray Requests and Associated Factors before Intervention}

In our sample, $48.69 \%$ (93/191) of lumbar spine X-ray requests were not relevant before the requesters training. The largest proportion of irrelevant requests came from the paramedical staff $(75.86 \%, \mathrm{p}<0.05)$ and requesters with more than 15 years of experience in medical practice $(76.71 \%)$. 
Table 1. Socio-demographic characteristics and distributions of the requesters.

\begin{tabular}{ccc}
\hline Variables & Number & Proportion (\%) \\
\hline Specialist doctor & 90 & 47.12 \\
General practitioner & 63 & 32.98 \\
Paramedic & 29 & 15.18 \\
Residents & 9 & 4.71 \\
& Number of years of experience & \\
$<5$ & 20 & 10.47 \\
{$[5-10[$} & 42 & 21.99 \\
{$[10-15[$} & 56 & 29.32 \\
$\geq 15$ & 73 & 38.22 \\
\hline
\end{tabular}

When considering the department, the patient was referred from, the proportion of irrelevant requests was higher in the private health facilities $(68.18 \%, \mathrm{p}<0.05)$ compared to the public health facilities.

Irrelevant requests were more common $(72.42 \%, \mathrm{p}<0.05)$ among patients themselves (Table 2).

\subsection{Effect of Training on the Appropriateness of X-Ray Request}

We selected 16 requesters for the intervention, and we compared the relevance of their X-ray requests before the intervention ( 79 requests) and 2 weeks after the intervention (45 requests). The overall proportion of irrelevant requests dropped from $58.22 \%$ (46/79) before training to $44.44 \%$ (21/45) after training, thus a reduction of $13.78 \%(\mathrm{p}<0.05)$ among the requesters who were trained.

The most important drop in the proportion of irrelevant requests was found among general practitioners (-21.98\%; Figure 1$)$ and requesters having less than 10 years of experience in medical practice ( $-31.8 \%$; Figure 2$)$. Conversely, there was a slight worsening in the group of paramedical personnel (-3.90\%).

\section{Discussion}

The objective of our study was to assess the effect of requester training on the relevance of lumbar spine X-ray requests for LBP in adults at Yaounde. The overall rate of irrelevant examinations was $48.69 \%$ before the training. This rate dropped from $58.22 \%$ (46/79) before training to $44.44 \%$ (21/45) after training $(-13.78 \%, \mathrm{p}=0.012)$ among the requesters who were trained. The highest drop after training was found among the requesters having less than 10 years of experience in medical practice $(-31.8 \%)$ and general practitioners $(-21.98 \%)$.

The request for irrelevant tests is common in medical practice. We found that half of the lumbar spine X-rays requested (48.69\%) were irrelevant and this inappropriate prescription rate was higher among paramedical staff (76.71\%). In a 


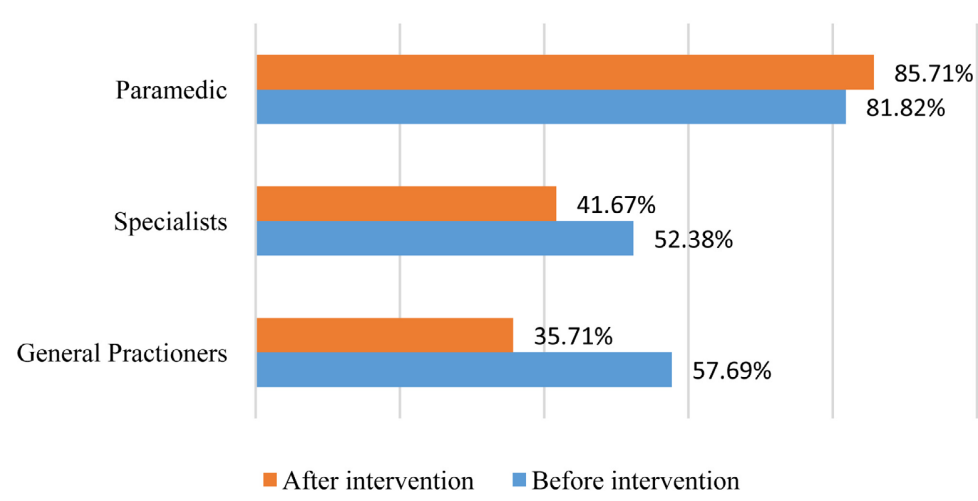

Figure 1. The proportion of irrelevant X-ray requests before and after the training according to the qualification of the requester.

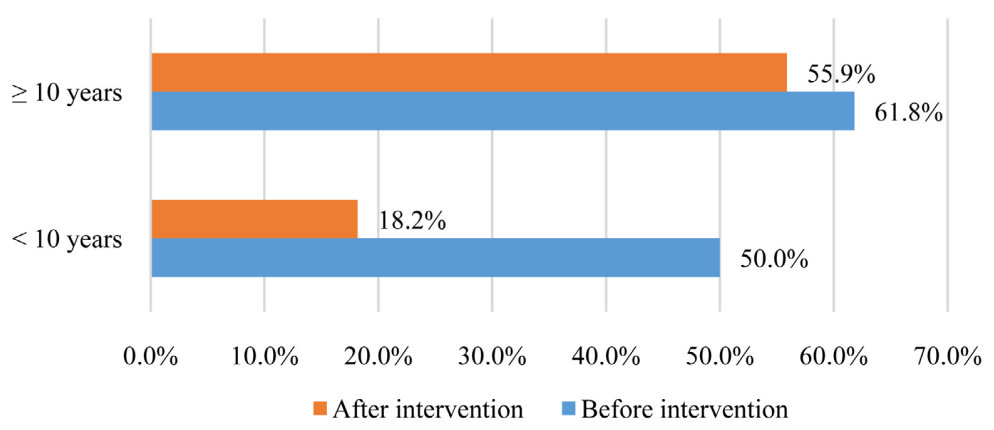

Figure 2. The proportion of irrelevant $\mathrm{X}$-ray requests before and after the training according to the number of years of experience of the requester.

Table 2. Factors associated with irrelevant X-ray requests.

\begin{tabular}{|c|c|c|c|}
\hline & \multicolumn{2}{|c|}{ Relevance of examination } & \multirow{2}{*}{$\mathrm{p}$} \\
\hline & Relevant: n (\%) & Irrelevant: $\mathrm{n}(\%)$ & \\
\hline \multicolumn{3}{|c|}{ Qualification of the requester } & \multirow{5}{*}{$<0.05$} \\
\hline General Practitioner & $34(53.97)$ & $29(46.03)$ & \\
\hline Residency Student & $4(44.44)$ & $5(55.56)$ & \\
\hline Specialist & $48(53.33)$ & $42(46.67)$ & \\
\hline Paramedic & $7(24.14)$ & $22(75.86)$ & \\
\hline \multicolumn{4}{|c|}{ Number of years of experience of the requester } \\
\hline$<5$ & $13(65)$ & $7(35)$ & \multirow{4}{*}{$\leq 0.001$} \\
\hline$[5-10[$ & $23(56.10)$ & $18(43.9)$ & \\
\hline$[10-15[$ & $40(71.43)$ & $16(28.57)$ & \\
\hline$\geq 15$ & $17(23.29)$ & $56(76.71)$ & \\
\hline \multicolumn{4}{|c|}{ Type of radiology center } \\
\hline Public hospital & $72(57.6)$ & $53(42.4)$ & $\leq 0.001$ \\
\hline Private facility & $21(31.82)$ & $45(68.18)$ & \\
\hline \multicolumn{4}{|c|}{ Type of requester } \\
\hline Patient himself & $8(27.58)$ & $21(72.42)$ & $<0.05$ \\
\hline Physician & $85(52.47)$ & $77(47.53)$ & \\
\hline
\end{tabular}


study of 5200 X-rays in Lome (2014), Adambounou et al. reported inappropriate prescriptions in $88 \%$ of cases among paramedical staff, $28 \%$ among general practitioners and 3.5\% among specialist physicians [10]. Their study was carried out in two public University Hospital Centers and involved X-rays of several anatomical parts. In Cameroon, paramedical staff are the most numerous healthcare workers and in the absence of systematic recruitment in the public sector, they work mostly in private practice where they perform medical consultations and do requests for radiology tests. However, their basic training prepares them to provide care and not to perform medical procedures; this may justify their lower compliance with the guidelines in radiology. Paramedical staff therefore represent one of the priority targets for improving the appropriateness of imaging examination requests.

The training of requesters is one of the main measures recommended to improve the relevance of requests for medical imaging examinations. In the United States, Schectman et al. had obtained a 5.4\% improvement in compliance with the guidelines on the management of acute low back pain after an intervention consisting of a training session and educational follow-up for requesters over one year [11]. Possible interventions to improve the adherence of requesters to guidelines are many: face-to-face training sessions, distribution of educational materials, evaluation of practices, and regular feedback to requesters, on-site practical training by experts [12]. These interventions have different results depending on the context and the profile of the clinicians. A combination of several of them would be more effective than implementing just one, and active methods such as face-to-face continuous educative sessions are more effective than passive methods such as the simple distribution of guidelines [8]. In our study, an educational discussion combined with the distribution of materials resulted in a significant improvement in the quality of prescriptions.

Despite an improvement of $13.78 \%$ due to the requesters training, the rate of irrelevant examinations after intervention remained relatively high (44.44\%, 20/45). Apart from ignorance of the guidelines, there are many reasons for irrelevant requests such as the expectation of financial incentives and insistent requests from the patients themselves ( 29 cases in our study). The policy of giving financial incentives to requesters has become a rule in private radiology practice in Cameroon. This is probably one of the reasons why irrelevant examinations are more frequently found in the private radiology department. In 2009, Pham et al. demonstrated, in a survey on the speed of prescribing imaging in elderly patients with low back pain, that practitioners paid for their cost-effectiveness, requested earlier and more often medical imaging examinations. Similarly, clinicians paid based on patient satisfaction, requested examinations early and requested more complex examinations (CT and MRI). Adequate remuneration of clinicians based on the quality of their professional practice rather than on profitability or patient satisfaction would significantly reduce requests for irrelevant tests [6] [8]. 
General practitioners $(+21.98 \%)$ and younger practitioners $(<10$ years of experience, $+68.89 \%)$ improved the relevance of their requests better than specialist physicians and more experienced practitioners. The latter were probably less receptive to the training of requesters and less opened to a change in their practice since they may estimate that their qualification and experience give them an expertise which should not be contested.

This study had some limitations that need to be stated. Our project would be more useful if it covered a wider population or tested a very novel innovative intervention. However, this preliminary study allows us to have a basic situation on which we can build an innovative training project adapted to our environment. Wider knowledge of how the improvements to the durability of training effects can be made would be useful, and this constitutes an interesting research perspective to sustainably improve the quality of exam requests.

\section{Conclusion}

Almost half of the X-rays prescribed for low back pain in adults at Yaounde are irrelevant. The highest proportion of irrelevant requests is found when the requester is a paramedical staff, when he has experience of more than 15 years, when the patient is referred to a private imaging center, and when the examination is requested by the patient himself. The training of requesters reduces the rate of irrelevant examinations moderately. Other factors of irrelevant prescriptions need to be sought and corrected for this reduction to be more significant.

\section{Acknowledgements}

To Mrs Atem Janice who revised the manuscript for language improvement.

\section{Conflicts of Interest}

The authors declare that they have no conflicts of interests.

\section{Funding Statement}

This research did not receive any specific grant from funding agencies in the public, commercial, or not-for-profit sectors.

\section{References}

[1] Agence Nationale d'Accréditation d'Evaluation en Santé (ANAES) (2000) Diagnostic, prise en charge et suivi des malades atteins de lombalgie chronique. Décembre; 2000.

[2] Fautrel, B., de Sauverzac, C., Rozenberg, S. and Bourgeois, P. (1998) Facteurs de risques professionnels d'origine biomécanique et physiologique et lombalgies. Revue du Rhumatisme-Edition Francaise, 65, 7S.

[3] Solange, D.M., Henry, L.N., Yone, E.W.P., Balkissou, A.D., Benjamin, T.N., Mathieu, M., et al. (2013) Présentation clinique et radiologique de la lombalgie chronique en consultation hospitalière à Douala-Cameroun. Journal Africain d Imagerie Médicale, $5,83-88$.

[4] Chou, R., Fu, R., Carrino, J.A. and Deyo, R.A. (2009) Imaging Strategies for Low-Back 
Pain: Systematic Review and Meta-Analysis. The Lancet, 373, 463-472. https://doi.org/10.1016/S0140-6736(09)60172-0

[5] Webster, B.S., Courtney, T.K., Huang, Y.-H., Matz, S. and Christiani, D.C. (2005) Physicians' Initial Management of Acute Low Back Pain Versus Evidence-Based Guidelines. Influence of Sciatica. Journal of General Internal Medicine, 20, 1132-1135. https://doi.org/10.1111/j.1525-1497.2005.0230.x

[6] Pham, H.H., Landon, B.E., Reschovsky, J.D., Wu, B. and Schrag, D. (2009) Rapidity and Modality of Imaging for Acute Low Back Pain in Elderly Patients. Archives of Internal Medicine, 169, 972-981. https://doi.org/10.1001/archinternmed.2009.78

[7] Moifo, B., Kamgnie, M.N., Fuh, F.N., Zeh, O.F., Tebere, H., Tapouh, J.R.M., et al. (2013) Pertinence of Indications of Medical Imaging Examinations at Yaounde-Cameroon. Health Sciences and Disease, 14.

[8] Chou, R. (2011) Diagnostic Imaging for Low Back Pain: Advice for High-Value Health Care from the American College of Physicians. Annals of Internal Medicine, 154, 181. https://doi.org/10.7326/0003-4819-154-3-201102010-00008

[9] Société Française de Radiologie (2013) Guide du bon usage des examens d'imagerie médicale. Editions SFR, Paris. http://gbu.radiologie.fr/

[10] Adambounou, K., Adjenou, K., Gbande, P., Agoda-Koussema, K., Jonhson, K., Dagbe, M., et al. (2014) Profil et évaluation de la justification des examens radiographiques standards réalisés dans les CHU de Lomé (TOGO). Journal Africain d'Imagerie Médicale, 5, 1-13.

[11] Schectman, J.M., Schroth, W.S., Verme, D. and Voss, J.D. (2003) Randomized Controlled Trial of Education and Feedback for Implementation of Guidelines for Acute Low Back Pain. Journal of General Internal Medicine, 18, 773-780. https://doi.org/10.1046/j.1525-1497.2003.10205.x

[12] French, S.D., Green, S., Buchbinder, R. and Barnes, H. (2010) Interventions for Improving the Appropriate Use of Imaging in People with Musculoskeletal Conditions. Cochrane Database of Systematic Reviews, 2010, CD006094.

https://doi.org/10.1002/14651858.CD006094.pub2 